\title{
Pierre Bouretz, Lumières du Moyen-Âge. Maïmonide philosophe
}

Paris, Gallimard, coll. « NRF Essai », 2015, 945 p.

Benjamin Fabre

\section{(2) OpenEdition}

\section{Journals}

Édition électronique

URL : http://journals.openedition.org/assr/28192

DOI : $10.4000 /$ assr.28192

ISSN : $1777-5825$

Éditeur

Éditions de l'EHESS

Édition imprimée

Date de publication : 31 décembre 2016

Pagination : 278

ISSN : 0335-5985

Référence électronique

Benjamin Fabre, «Pierre Bouretz, Lumières du Moyen-Âge. Maïmonide philosophe », Archives de sciences sociales des religions [En ligne], 176 | octobre-décembre 2016, mis en ligne le 17 juillet 2017, consulté le 23 septembre 2020. URL : http://journals.openedition.org/assr/28192 ; DOI : https:// doi.org/10.4000/assr.28192

Ce document a été généré automatiquement le 23 septembre 2020.

(c) Archives de sciences sociales des religions 


\section{Pierre Bouretz, Lumières du Moyen- Âge. Maïmonide philosophe}

Paris, Gallimard, coll. « NRF Essai », 2015, 945 p.

\section{Benjamin Fabre}

\section{RÉFÉRENCE}

Pierre Bouretz, Lumières du Moyen-Âge. Maïmonide philosophe, Paris, Gallimard, coll. «NRF Essai », 2015, 945 p.

1 On ne conseillera pas seulement la lecture de cet ouvrage imposant à des spécialistes ou à des érudits. Le lecteur rencontrera là en effet une problématique qui n'a pas cessé de traverser l'histoire de la pensée, et particulièrement de la pensée religieuse. Comment concilier les avancées, disons les conquêtes, de la philosophie et le socle de la Révélation? Peut-on conserver la fidélité à celle-ci sans renoncer tout simplement à penser? Les tentatives d'une conciliation peuvent-elles soutenir le croyant, ou conduire inéluctablement et après quelques acrobaties intellectuelles à l'abandon d'une foi traditionnelle? Maïmonide au XII ${ }^{\mathrm{e}}$ siècle nous pose pleinement ces interrogations. Que ce soit à l'intérieur de la seule tradition juive ne diminue en rien leur intérêt pour tout croyant - et pour tout penseur.

2 On s'étonnera de voir la biographie de Maïmonide évoquée en note, l'auteur invoquant l'impossibilité même de l'esquisser. Il serait cependant utile d'en savoir un peu plus sur les trajets et les contextes de ce philosophe tout de même assez mal connu en général. On ne voit pas bien pourquoi il serait impossible d'y parvenir, la note en question soulevant au contraire les nombreux éléments qui le permettraient. Dans le cours de l'ouvrage, on aurait souvent envie de s'y référer un peu plus précisément. Puisque nous parlons de notes, j'insisterais sur l'importance de celles-ci dans le travail de Pierre Bouretz. Elles occupent la moitié de ce gros ouvrage de plus de 900 pages. Elles témoignent en tous cas d'une incroyable érudition de l'auteur qui semble maitriser toute la littérature consacrée à la pensée du haut Moyen-Âge. Beaucoup de ces notes 
sont absolument indispensables pour une bonne entente de la pensée de Maïmonide, et leur intégration au texte même serait souvent souhaitable.

Bouretz rappelle en introduction que l'Antiquité n'a évidemment pas connu le conflit théologico-philosophique qui devient au contraire « déterminant » pour la philosophie médiévale, aussi bien chez les juifs, que chez les chrétiens et plus tard les arabes. Quelque chose existe alors qui est comme le foyer de l'effort théorique des penseurs : ce que Maïmonide nomme «la perplexité ». Maïmonide décrit ainsi son lecteur : «celui qui a étudié la philosophie et qui a acquis des sciences véritables, mais qui, croyant aux choses de la loi, est perplexe au sujet de leur sens. » Tous les déterminants du champ philosophique de Maïmonide sont réunis là : l'étude, la science, la croyance, la loi, et la perplexité dont il faut tout de même essayer de sortir autant qu'il est possible. Léo Strauss en 1935 est le premier à avoir montré que cette problématique issue pour nous des Lumières s'imposait bien avant notre époque moderne et qu'on peut parler de Lumières médiévales dont Maïmonide est l'un des protagonistes essentiels : quels tourments les Lumières ont-elles alors déjà infligés à la Foi ?

4 Bouretz expose tout de suite l'importance qu'a eue - malgré les deux siècles qui les séparent - pour Maïmonide, la pensée du philosophe arabe al Farabi (870-950), auquel d'ailleurs il se réfère tout au long de son étude. Pour al Farabi la philosophie est en grave danger. Ou plutôt elle serait en une grave situation, accusée avec raison d'élitisme et d'ésotérisme, si son intention n'était pas " politique par nature », c'est dire que tout à l'opposé d'une délectation aristocratique elle a pour finalité de "vivre en harmonie avec le vulgaire [...] d'améliorer sa condition [...] de lui faire percevoir la vérité dans les opinions religieuses ». Ce qui revient à supposer que cette vérité n'est pas forcément perçue ni perceptible au sein de ces opinions, et qu'il faut donc donner au «vulgaire» la possibilité de les percevoir au moyen de l'exercice philosophique. L'un des secrets les mieux gardés de la philosophie médiévale, dira Bouretz (p. 48), c'est l'idée d'une philosophie populaire reliant Aristote à Platon. Ce n'est pas tout d'être sorti de la caverne, il faut y redescendre et c'est la pensée d'Aristote qui le permettra. La croyance religieuse peut devenir un obstacle à la Vérité si elle reste confuse et en quelque sorte impensée. "Il arrive que l'homme aime les opinions qui lui sont familières et dans lesquelles il a été élevé, qu'il les protège et s'effraie de ce qui est loin d'elles.» Toute habitude peut être pernicieuse. Mais jusqu'où aller dans ce travail critique ? Par exemple, pour Maïmonide, la croyance en la corporéité de Dieu doit être totalement éradiquée. Elle provient de ce fait que «tout ce qui n'est pas un corps n'a pas pour les hommes d'existence ». Soit. Mais on ne doit pas pour autant mettre en péril la croyance en un Dieu qu'on ne peut plus par ailleurs « représenter ». Dieu luimême dans la Révélation, agit avec ruse et n'impose pas à ses fidèles la brutalité d'une transcendance totalement abstraite... Il faudra donc beaucoup de prudence dans la mise en œuvre philosophique, et toujours considérer à qui le discours s'adresse, pour qui il est fécond et pour qui il ne le serait pas. La pensée de Maïmonide sera régie par ce que Bouretz appelle "un principe d'accommodation ». Ce qui aura nécessairement pour effet de produire la subtilité, mais aussi souvent l'ambiguïté voire les obscurités de son propos.

5 On aura ainsi trois types d'arguments selon les capacités de compréhension propres à différentes catégories d'individus : rhétoriques s'agissant de tous, dialectiques pour les hommes de la loi, démonstratifs pour l'élite spirituelle. Ainsi faudra-t-il toujours nier la corporéité de Dieu, même si on n'est pas toujours en mesure de dire pourquoi et de 
démontrer son impossibilité. Ce que le plus grand nombre pourra comprendre, c'est l'incommensurabilité entre Dieu et l'homme. Pourra-t-il toujours aller jusqu'à penser la nature divine au-delà de cette négation d'une imagerie naïve ? Ce n'est pas sûr, et il ne faut pas risquer ici d'aller trop vite et trop loin. Mais dit Maïmonide, «j'aurais cru commettre une grande lâcheté envers tout homme perplexe en m'abstenant de rien écrire de ce qui est clair pour moi.» Maïmonide se partage entre sa responsabilité envers le grand nombre et une contemplation solitaire qui reste la plus haute finalité de la pensée.

6 J'ai évoqué un peu plus haut les hommes de la loi. La réflexion sur la loi et ses interprètes occupe une grande place dans la pensée de Maïmonide. Entre le vulgaire et l'élite, Bouretz définit les hommes de la loi comme ceux qui sont en charge de faire respecter les dogmes et les pratiques, «étant ainsi les parents de la religion » (p. 90). À eux de veiller sur l'observance « des règles qui concernent les relations des hommes entre eux et le bien-être de la société ». Ces hommes, selon Maimonide, admettent par tradition les opinions vraies, mais ne s'engagent pas «dans la spéculation sur les principes fondamentaux de la religion ». Ce sont moins des théologiens qu'en somme des positivistes du religieux. C'est eux qu'il faut aider à s'élever au-dessus d'une compréhension traditionnelle des vérités de la loi. Aux yeux de Bouretz, il y a cependant quelque ambiguïté quant aux intentions de Maïmonide rédigeant pour ces hommes la "Misnah Torah ». Il s'agit de simplifier et rendre clair le contenu de la loi, de mettre en lumière les éléments en quelque sorte naturels de la loi, dans la tradition aristotélicienne d'al Farabi par exemple, mais aussi d'éveiller la conscience du caractère non naturel de la loi, de ses liens avec la fonction du prophète, conçu comme le médiateur de l'Intellect divin législateur. Mais cette fonction prophétique, et même dans le cas de Moïse, est aussi considérée comme «l'exercice d'une faculté naturelle» : «tout ce qui peut être su par le raisonnement l'est absolument au même titre par le prophète et par tout autre qui le sait, sans qu'il y ait là une supériorité de l'un sur l'autre. » Le champ prophétique est ainsi, selon Bouretz, considérablement restreint, sans toutefois supprimer la tension entre l'épanchement divin et l'activité humaine qu'il favorise. Le prophète n'est pas un contemplatif occupé seulement de la pensée de Dieu, il veut guider et instruire les habitants de la Terre et par là il se rapproche du philosophe pour être avec lui une figure de l'excellence.

7 Si l'Écriture parle le langage des hommes, selon Maïmonide, en s'adaptant à leurs possibilités de compréhension, on ne peut évidemment pas s'adresser à eux dans un langage qui ne serait plus humain. L'autre langage auquel Maïmonide s'efforce dans le Guide des perplexes de parvenir, doit assumer la possibilité d'une autre écoute humaine possible, celle d'hommes dont le niveau spirituel aurait suffisamment évolué pour leur permettre de l'entendre. Il s'agit bien de faire en sorte que tous puissent parvenir à cette nouvelle entente. C'est l'objectif du Guide des perplexes, à travers la mise en question de questions théologiques essentielles.

8 Le problème de l'origine du monde est pour Maïmonide une question si centrale qu'il peut affirmer que «tout dépend de cette question ». C'est l'affrontement déterminant des philosophes et des théologiens - mais Bouretz montre là l'attitude fondamentale de Maïmonide. On peut souhaiter le point de vue des théologiens avec force raisons, mais dit Maïmonide, «le plus grand hommage que j'aie pu rendre à la vérité, c'est d'avoir ouvertement déclaré combien ces matières me jetaient dans la perplexité et que je n'avais ni entendu ni connu de démonstrations pour aucune d'elles »: perplexité du 
Guide des perplexes, ouverture à la nécessité d'une contemplation toujours en quête d'une vérité peut-être inaccessible. Il semble que Maïmonide ait moins le projet d'opposer une théologie à une autre que celui de les dépasser toutes par un souci inextinguible de la Vérité. Bouretz en vient à qualifier de tortueuses les démarches philosophiques de Maïmonide, à parler de méandres, et ce n'est pas le moindre mérite de son livre que de rendre clair un cheminement qui se veut à la fois prudent et loin de tout compromis. Que pense vraiment Maïmonide? En ce qui concerne les problèmes classiques de la philosophie, il s'agit moins de les résoudre que d'ébranler les discours dans le souci d'une Vérité jamais formulée. On pourrait parler, comme le fait Bouretz, d'un scepticisme « critique » avec six siècles d'avance. 